\title{
A Model of Optimal Development for an Economy with an Informal Sector
}

\author{
Prabir C. Bhattacharya \\ Department of Accountancy, Economics, and Finance, Heriot-Watt University, Edinburgh, UK \\ Email: p.c.bhattacharya@hw.ac.uk
}

How to cite this paper: Bhattacharya, P.C. (2017) A Model of Optimal Development for an Economy with an Informal Sector. Journal of Applied Mathematics and Physics, 5, 1808-1824.

https://doi.org/10.4236/jamp.2017.59153

Received: August 11, 2017

Accepted: September 24, 2017

Published: September 27, 2017

Copyright $\odot 2017$ by author and Scientific Research Publishing Inc. This work is licensed under the Creative Commons Attribution International License (CC BY 4.0).

http://creativecommons.org/licenses/by/4.0/

\begin{abstract}
The paper addresses the question of optimal development of a developing economy. The framework presented, it is believed, can be of help in thinking about policies relating, inter alia, to population growth, inter-sectoral migration, agriculture-industry relationship, wages in different sectors, and income distribution in an inter-connected way in the context of optimal development of an economy with an informal sector.
\end{abstract}

\section{Keywords}

Optimal Development, Informal Sector, Income Distribution, Numerical Examples

\section{Introduction}

The aim of this paper is to address the question of optimal development of a developing economy with an informal sector. The problem of planned optimal development in a dual economy-with the economy sectorised into an advanced or modern and a traditional or rural sector-was investigated in the literature in the 1960s and 1970s (see, among others, [1] [2] [3]). That literature assumed that the advanced sector of the economy is fully planned so that it is possible for the planners to control the level of employment as well as consumption directly. It was further assumed that the government has direct control over the process of rural-urban migration. It was then shown that under such conditions there exists a unique optimal growth path that will take the economy from any initial state to a unique steady-state.

However, today it seems unreasonable to assume that the modern sector of the economy is or can be fully planned. Instead, it would appear more appropriate to assume that while the planners can have control over the wage in the ad- 
vanced sector-which we shall call the formal sector below-through wage legislation, the producers in that sector fix employment by profit maximization. It would also appear reasonable to assume that the government has no direct control over the process of rural-urban migration.

In recent years, the informal sector has become an important focus of research and in the presence of an informal sector, the economy can be sectorised into rural, informal and formal sectors (with the last two sectors being assumed to be located in the urban area). ${ }^{1}$ Empirical evidence increasingly suggests that an overwhelmingly large number of migrants from the rural to the urban area migrate to the informal sector and without any thoughts of obtaining jobs in the formal sector; further that there is very little job search activity by the migrant workers in the informal sector. ${ }^{2}$ In the analysis below we shall, therefore, assume, for simplicity, that people not born in the urban area do not get formal sector jobs or believe that they can get such jobs.

Our aim in this paper is to model such an economy to investigate the existence of a steady-state and to study the properties of the optimal path. We shall use numerical examples to illustrate the model.

\section{The Model and the Steady-State}

We take a one-good economy. Output in the formal sector is $h(K, F)$, where $h$ is linear homogeneous, $K$ and $F$ are formal sector capital stock and employment, respectively. Both the rural and informal sector outputs are produced with labour alone. $L$ is the total labour in the urban area (consisting of workers employed in the formal and informal sectors).

The migration function is given by $F_{m}(v-w)(1-l)$, where $v$ is the informal sector wage and $w$ the rural sector wage. That is, the proportion of rural population who migrate from the rural to the urban area is a function of the difference between $v$ and the rural sector wage, $w$, and the greater the difference, the greater will be this proportion. ${ }^{3}$ The informal sector wage, $\mathrm{V}$, is positively related to the number of workers in the formal sector ${ }^{4}$ and negatively related to the number of workers in the urban area. The rural sector wage, $w$, is negatively related to the number of workers in the rural area. We shall employ two parameters $\gamma$ and $\beta$ to reflect the demand for labour in the informal and rural sec${ }^{1}$ For a model of a developing economy that systematically incorporates an informal sector, see [7] [8].

${ }^{2}$ See, for example, [7] [9] [10] [11]. These empirical findings are in contradiction to the basic assumptions underlying the [12] and [13]-type models. As [14] noted in his article in the Handbook o1 Development Economics, “Todaro's job-lottery and high unemployment view of the labour market in the Third World simply fails to pass the test of evidence".

${ }^{3}$ There are both psychological and other costs involved in migration, and while some rural dwellers will migrate if $v$ is marginally higher than $w$, others, perhaps of less adventurous spirit or more attuned to the rural way of life, would be motivated to migrate only if the difference between $v$ and $w$ is very much greater. Potential migrants, in other words, have different levels of inertia in the face of a given difference between $v$ and $w$, so that the greater the difference between $v$ and $w$ the greater will be the proportion of rural dwellers who would actually migrate.

${ }^{4} \mathrm{~A}$ greater number of workers in the formal sector will, ceteris paribus, tend to decrease the labour supply to the informal sector and so tend to increase the informal sector wage, $v$. 
tors, respectively. (We shall argue later in Section 4 that these parameters are susceptible to influences by government policies). We assume that all wages are consumed and all formal sector profit is invested. ${ }^{5}$

The planners fix the formal sector wage rate, $v^{*}$. The producers in the formal sector fix employment by profit maximization. Hence $v^{*}=h_{f}(k, f)$ so that $f=g\left(k, v^{*}\right)$. (The lower case letters denote the corresponding upper case variables divided by population $N$. We shall henceforth talk in terms of such intensive variables only). To ensure that the formal sector labour demand is satisfied, we assume that $v^{*} \geq \alpha v$, where $\alpha \geq 1$.

With the formal sector wage, $v^{*}$, as the control variable, we have the following planning problem:

$$
\max \int_{0}^{\infty}\left[f u\left(v^{*}\right)+(l-f) u(v)+(1-l) u(w)\right] \mathrm{e}^{-\delta t} \mathrm{~d} t
$$

(where $u$ is the individual's utility function (which depends on income alone); $\delta=\phi-n>0, \phi$ being the rate of discount and $n$ the exponential rate of population growth).

The problem is defined by the Hamiltonian

$$
\begin{aligned}
H= & f u\left(v^{*}\right)+(l-f) u(v)+(1-l) u(w) \\
& +\lambda_{k}\left[h(k, f)-v^{*} f-n k\right] \\
& +\lambda_{l}\left[F_{m}(v-w)(1-l)\right]
\end{aligned}
$$

The state variables $l$ and $k$ are governed by the equations:

$$
\begin{gathered}
\dot{k}=h(k, f)-v^{*} f-n k \\
\dot{l}=F_{m}(v-w)(1-l)
\end{gathered}
$$

The control variable $v^{*}$ must obey the constraint: $v^{*} \geq \alpha v$ with $v=\frac{\gamma f}{l}$ and $w=\frac{\beta}{1-l}$. In the numerical example, we take $h(k, f)=k^{\frac{1}{3}} f^{\frac{2}{3}}$ and $u(c)=-\frac{1}{c} \cdot{ }^{6}$ Knowing that $h_{f}=v^{*}$ and $h_{f}=\frac{2}{3}\left(\frac{k}{f}\right)^{\frac{1}{3}}$, we can rewrite $v$ in function of $v^{*}: v=\frac{8 \gamma k}{27 l v^{* 3}}$. Thus the condition $v^{*} \geq \alpha v$ can be rewritten as $v^{*} \geq\left(\frac{8 \alpha \gamma k}{27 l}\right)^{\frac{1}{4}}$. Therefore, the Hamiltonian can be expressed as

\footnotetext{
${ }^{5}$ Capital accumulation, it will be recalled, is assumed to take place only in the formal sector.

${ }^{6} \mathrm{We}$ are assuming production to be Cobb-Douglas and the utility function we use means that we value an extra unit of consumption accruing to a particular group, say, group A four times as much as a unit accruing to another group B if group B has consumption per head twice as large as group A. It would, of course, be possible to work with other utility functions. For interest, we did some exercises using $u(c)=\log _{\mathrm{e}} c$. The results in these cases turned out to be not that different from those obtained by using $u(c)=-\frac{1}{c}$.
} 


$$
\begin{aligned}
H= & -\frac{8 k}{27 l v^{* 4}}-\frac{\left(l-\frac{8 k}{27 * v^{* 3}}\right) 27 l v^{* 3}}{8 \gamma k}-\frac{(1-l)^{2}}{\beta} \\
& +\lambda_{k}\left[\frac{4 k}{9 v^{* 2}}-\frac{8 v^{*} k}{27 v^{* 3}}-n k\right]+\lambda_{l}\left[F_{m}\left(\frac{8 \gamma k}{27 l v^{* 3}}-\frac{\beta}{1-l}\right)(1-l)\right]
\end{aligned}
$$

Or, with reducing what can be reduced:

$$
\begin{aligned}
H= & -\frac{8 k}{27 v^{* 4}}-\left(\frac{27 l^{2} v^{* 3}}{8 \gamma k}-\frac{l}{\gamma}\right)-\frac{(1-l)^{2}}{\beta}+\lambda_{k}\left[\frac{4 k}{27 v^{* 2}}-n k\right] \\
& +\lambda_{i}\left[F_{m}\left(\frac{8 \gamma k}{27 v^{* 3}}\left(\frac{1}{l}-1\right)-\beta\right)\right]
\end{aligned}
$$

Thus the Lagrangian is, in function of $v^{*}$ :

$$
\Phi=H+m\left[v^{*}-\left(\frac{8 \alpha \gamma k}{27 l}\right)^{\frac{1}{4}}\right]
$$

The differential calculations to maximize the Hamiltonian lead to:

$$
\begin{gathered}
\frac{\partial H}{\partial k}=-\frac{8}{27 v^{* 4}}+\frac{27 l^{2} v^{* 3}}{8 \gamma k^{2}}+\lambda_{k}\left[\frac{4}{27 v^{* 2}}-n\right]+\lambda_{l}\left[\frac{8 F_{m} \gamma}{27 v^{* 3}}\left(\frac{1}{l}-1\right)\right] \\
\frac{\partial H}{\partial l}=-\left(\frac{27 l v^{* 3}}{4 \gamma k}-\frac{1}{\gamma}\right)+\frac{2(1-l)}{\beta}-\frac{8 \lambda_{l} \gamma F_{m} k}{27 v^{* 3} l^{2}}
\end{gathered}
$$

Therefore, using $\frac{\partial H}{\partial k}=-\left(\dot{\lambda}_{k}-\delta \lambda_{k}\right)$ and $\frac{\partial H}{\partial l}=-\left(\dot{\lambda}_{l}-\delta \lambda_{l}\right)$, where $\dot{\lambda}_{k}, \dot{\lambda}_{l}$ are the temporal derivatives of $\lambda_{k}, \lambda_{l}$ respectively, we obtain:

$$
\begin{gathered}
\dot{\lambda}_{k}=\frac{8}{27 v^{* 4}}-\frac{27 l^{2} v^{* 3}}{8 \gamma k^{2}}+\lambda_{k}\left[n+\delta-\frac{4}{27 v^{* 2}}\right]+\frac{8 \lambda_{l} F_{m}}{27 v^{* 3}}\left(1-\frac{1}{l}\right) \\
\dot{\lambda}_{l}=\frac{27 l v^{* 3}}{4 \gamma k}-\frac{1}{\gamma}+\frac{2(l-1)}{\beta}+\lambda_{l}\left[\frac{8 F_{m} \gamma k}{27 v^{* 3} l^{2}}+\delta\right]
\end{gathered}
$$

For the Lagrangian we have:

$$
\frac{\partial \Phi}{\partial v^{*}}=\frac{8 k}{27 v^{* 3}}\left[\frac{4}{v^{* 2}}-\frac{3\left(\frac{3}{2}\right)^{6} l^{2} v^{* 5}}{\gamma k^{2}}-\lambda_{k}-\frac{3 \lambda_{l} F_{m} \gamma k}{v^{*}}\left(\frac{1}{l}-1\right)\right]+m=0
$$

The governing differential equations of the state variables $k, l$ are now:

$$
\begin{gathered}
\dot{k}=\frac{4 k}{27 v^{* 2}}-n k \\
\dot{l}=F_{m}\left(\gamma f\left(\frac{1}{l}-1\right)-\beta\right)
\end{gathered}
$$

The stationary values of $\lambda_{k}, \lambda_{l}, l, k$ can be obtained as follows: 


$$
\begin{gathered}
\dot{\lambda}_{k}^{\text {stat }}=\frac{8}{27 v^{* \text { stat } 4}}-\frac{27 l^{\text {stat } 2} v^{* \text { stat } 3}}{8 \gamma k^{\text {stat } 2}}+\lambda_{k}^{\text {stat }}\left[n+\delta-\frac{4}{27 v^{\text {stat } 2}}\right] \\
+\frac{8 \lambda_{l}^{\text {stat }} \gamma F_{m}}{27 v^{* \text { stat } 3}}\left(1-\frac{1}{l^{\text {stat }}}\right)=0 \\
\dot{\lambda}_{l}^{\text {stat }}=\frac{27 l^{\text {stat }} v^{\text {*stat } 3}}{4 \gamma k^{\text {stat }}}-\frac{1}{\gamma}+\frac{2\left(l^{\text {stat }}-1\right)}{\beta}+\lambda_{l}^{\text {stat }}\left[\frac{8 F_{m} \gamma k^{\text {stat }}}{27 v^{* \text { stat } 3} l^{\text {stat } 2}}+\delta\right]=0 \\
\dot{k}^{\text {stat }}=\frac{4 k^{\text {stat }}}{27 v^{* \text { stat } 2}-n k^{\text {stat }}=0} \\
\dot{l}^{\text {stat }}=F_{m}\left(\gamma f^{\text {stat }}\left(\frac{1}{l^{\text {stat }}}-1\right)-\beta\right)=0
\end{gathered}
$$

From here it is easy to extract:

$$
\begin{gathered}
v^{* \text { stat }}=\frac{2}{9} \sqrt{\frac{3}{n}} \\
l^{\text {stat }}=\frac{\gamma f^{\text {stat }}}{\gamma f^{\text {stat }}+\beta} \\
\lambda_{l}^{\text {stat }}=\frac{\left(\frac{1}{\gamma}-\frac{27 l^{\text {stat }} v^{* \text { stat } 3}}{4 \gamma k^{\text {stat }}}-\frac{2\left(l^{\text {stat }}-1\right)}{\beta}\right)}{\frac{8 F_{m} \gamma k^{\text {stat }}}{27 v^{* \text { stat } 3} l^{\text {stat } 2}}+\delta} \\
\lambda_{k}^{\text {stat }}=\frac{\left(\frac{87 l^{\text {stat } 2} v^{* \text { stat } 3}}{8 \gamma k^{\text {stat } 2}}-\frac{8 \lambda_{l}^{\text {stat }} F_{m} \gamma}{27 v^{* \text { stat } 4}}-\frac{27 v^{\text {stat } 3}}{n+\delta-\frac{4}{27 v^{* \text { stat } 2}}}\left(1-\frac{1}{l^{\text {stat }}}\right)\right)}{n+\delta}
\end{gathered}
$$

We now assume that $m=0$, which is equivalent to $v^{*}>\left(\frac{8 \alpha \gamma k}{27 l}\right)^{\frac{1}{4}}$. In such case, we need to calculate $k^{\text {stat }}$ from the equation $\frac{\partial H}{\partial v^{*}}=0$, i.e., when we have used the expression of the stationaries written above:

$$
\frac{4}{v^{* \text { stat } 2}}-\frac{3\left(\frac{3}{2}\right)^{6} l^{\text {stat } 2} v^{* \text { stat } 5}}{\gamma k^{\text {stat } 2}}-\lambda_{k}^{\text {stat }}-\frac{3 \lambda_{l}^{\text {stat }} F_{m} \gamma k^{\text {stat }}}{v^{{ }^{\text {stat }}}}\left(\frac{1}{l^{\text {stat }}}-1\right)=0
$$

Thus we have an equation for $k^{\text {stat }}$ that depends only on $v^{* \text { stat }}$ and $l^{\text {stat }}$. These last two stationaries can be directly obtained from the problem parameters; we can therefore solve numerically the equation above (for example with a Newton-Raphson algorithm) to obtain $k^{\text {stat }}, \lambda_{l}^{\text {stat }}$ and $\lambda_{k}^{\text {stat }}$.

In case of $m>0$, which corresponds to $v^{*}$ being constrained, we can write $v^{* \text { stat }}=\left(\frac{8 \alpha \gamma k}{27 l}\right)^{\frac{1}{4}}$. In such scenario we obtain: 


$$
\begin{aligned}
k^{\text {stat }} & =\frac{2 l^{\text {stat }}}{27 \alpha \gamma n^{2}} \\
l^{\text {stat }} & =\frac{\left(\frac{2}{9 \alpha} \sqrt{\frac{3}{n}}-\beta\right)}{\frac{2}{9 \alpha} \sqrt{\frac{3}{n}}}
\end{aligned}
$$

The other stationary values can be then calculated from these.

The steady state solution (as well as the transient) to the maximization problem has to satisfy certain restrictions, namely, $0<k^{\text {stat }}$ and $0<l^{\text {stat }}<1$. These conditions are equivalent to $0<l^{\text {stat }}<1$ which after some rearrangements leads to $\beta<\frac{2}{9 \alpha} \sqrt{\frac{3}{n}}=\beta_{\max }$.

We have obtained the stationaries in the two cases where the condition on $v^{*}$ is binding or not. In addition, it can be observed that $v^{\text {stat }}=\frac{\gamma f^{\text {stat }}}{l^{\text {stat }}}=\gamma f^{\text {stat }}+\beta$ and $w^{\text {stat }}=\frac{\beta}{1-l^{\text {stat }}}=\gamma f^{\text {stat }}+\beta$. Therefore, at the steady state solution $v^{\text {stat }}=w^{\text {stat }}=\gamma f^{\text {stat }}+\beta$.

\section{The Optimal Path}

The optimal path can be obtained by maximizing the utility integral (discretizing the ordinary differential equations (ODEs) with a fourth order Runge-Kutta scheme) or by using the Hamiltonian where, in the application considered below, the coupled system of ODEs is discretized with an adaptive fourth-fifth order Runge-Kutta method ${ }^{7}$. The parameters that describe the behaviour of the model are $\alpha, \beta, \gamma, F_{m}, n$ and $\delta$. In what follows we first present results obtained with a particular set of these parameter values and initial conditions. Then in the next section we show how the effects of changes in these parameter values can be studied in a convenient way within the framework presented here.

The set of parameter values and initial conditions we use in this section are in Table 1.

Brief justifications for using these parameter values as a starting point are as follows 8 . We assume that a large number of people are available for work in the formal sector and that any premium that the formal sector employers may need

\footnotetext{
${ }^{7}$ A fourth-order fixed-step explicit Runge-Kutta method is chosen to discretize the set of differential equations. This selection owes to the proven numerical stability of the method and also to the relatively low error ( $O\left(h^{4}\right)$, where $h$ is the step size) for the computational cost of each iteration. The reason for not choosing a variable step methodology is that in this case to solve the optimization problem, the computational cost increases significantly, since to calculate the integral of the minimization problem a large number of points were required to reduce the errors. Therefore, a variable step methodology would be required to have a certain maximum step size, thus making the variable step size algorithm redundant.

${ }^{8}$ For fuller justifications of the parameter values and the initial conditions used than provided here, the interested reader is referred to the analysis in [8] [10]. Many of the parameter values and initial conditions used here are also within the range of values used in previous simulation studies in the literature.
} 
to pay to attract workers to that sector is, therefore, likely to be very small, and accordingly we have set $\alpha=1.1$. We have set the value of $\gamma$ higher than that of $\beta$. These parameters, it will be recalled, are included to reflect the demand for labour in the informal and rural sectors, respectively. These demands, in the interpretation adopted in this paper, are dependent on government policies (this is elaborated in Section 4 below). It is assumed that the relevant government policies can be more effectively pursued and implemented in the urban than in the dispersed and remote rural areas. We also assume the rate of discount (i.e., $\phi$ ) to be marginally higher than the exponential rate of population growth, and we have set $\delta=0.01$. However, as already mentioned, the effects of assuming different parameter values to those assumed here can be easily studied within the framework presented here and this question is addressed in the next section.

Using the parameter values specified in Table 1, the stationary solution is in Table 2.

It is also ascertained that at the steady state we have about 60 percent of workers in the formal sector, 10 percent in the informal sector, and 30 percent in the rural sector. Graphs tracing the paths of the formal sector wage, the informal sector wage, capital and urban labour force are shown in Figures 1-4, respectively. We have also computed the evolution of the Gini coefficient along the optimal path (Figure 5). It will be noted that the values of the Gini coefficient are very low. This is not surprising: the steady state values of the formal, informal, and rural sector wages are, as we have seen, 2.4 and 2.2, respectively. The wage differences are thus really small, not even a 10 percent. (The average wage is approximately 2.3 which means that the difference between the average and the extremes is less than 5 per cent). Nevertheless, despite the low values of the Gini coefficient, the evolution of the Gini coefficient traces out a path that looks somewhat like an inverted U-shaped curve. It was, of course, the contention of [4] that income inequality first increases and then declines with economic development and a large literature has since been devoted to exploring this and related issues. ${ }^{9}$ It is also clear that with the set of parameter values used, it takes a very long time for the steady state to be achieved. ${ }^{10}$ It is, therefore, of some interest to note that much earlier, [5] too, in the context of a discussion about the timescale of the neo-classical growth model, found the time required for the

Table 1. Parameter values and initial conditions.

\begin{tabular}{cccccccc}
\hline$\alpha$ & $\beta$ & $\gamma$ & $\delta$ & $F_{m}$ & $n$ & $k_{0}$ & $l_{0}$ \\
\hline 1.1 & 0.664 & 2.5 & 0.01 & 0.1 & 0.025 & 0.5 & 0.3 \\
\hline
\end{tabular}

Table 2. Stationary solution.

\begin{tabular}{cccccc}
\hline$\lambda_{l}^{\text {stat }}$ & $\lambda_{k}^{\text {stat }}$ & $v^{\text {stat }}$ & $v^{\text {stat }}$ & $k^{\text {stat }}$ & $l^{\text {stat }}$ \\
\hline 1.2264 & 0.4747 & 2.4343 & 2.213 & 30.1665 & 0.7 \\
\hline
\end{tabular}

${ }^{9}$ See, among others, [15] [16] [17] [18].

${ }^{10} \mathrm{We}$ have purposely not defined the X-coordinates of Figures 1-5 to allow for the degree of flexibility prevailing in different economies at different times, but a time unit of, say, half-a-year would probably capture the time span that we have in mind in the context of the economy that we have modelled. 


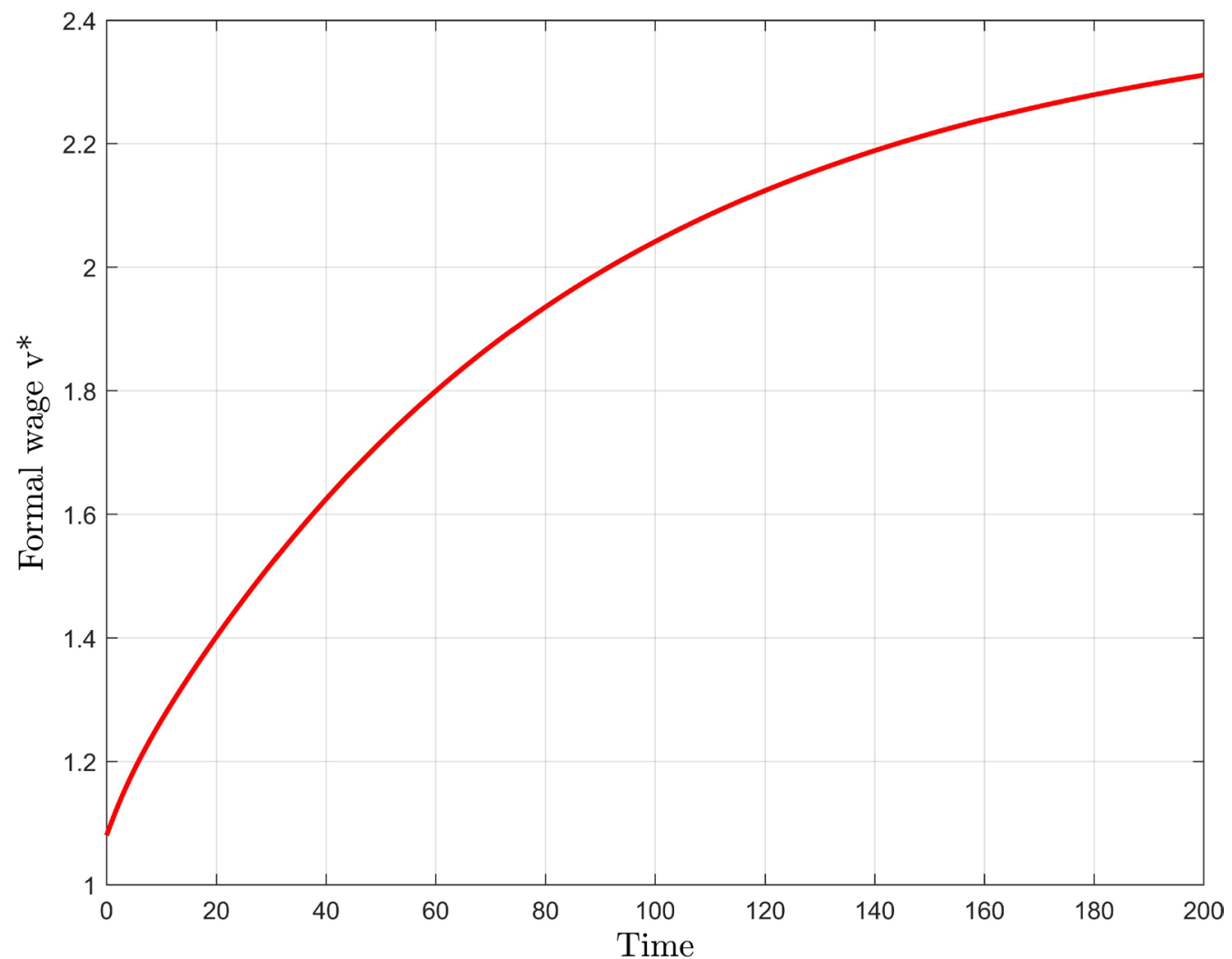

Figure 1. The optimal path of the formal sector wage, $v^{*}$.

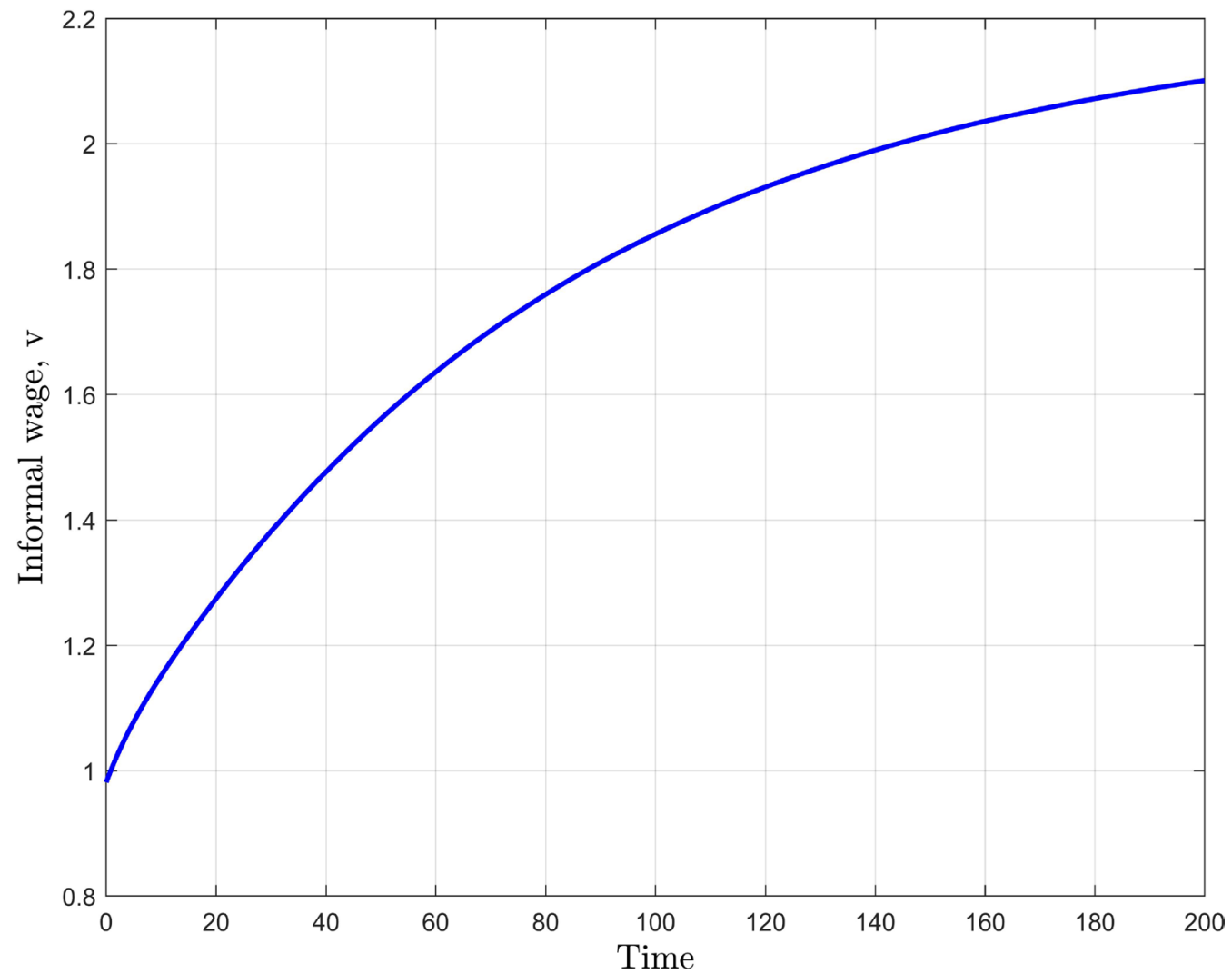

Figure 2. The path of the informal sector wage, $v$. 


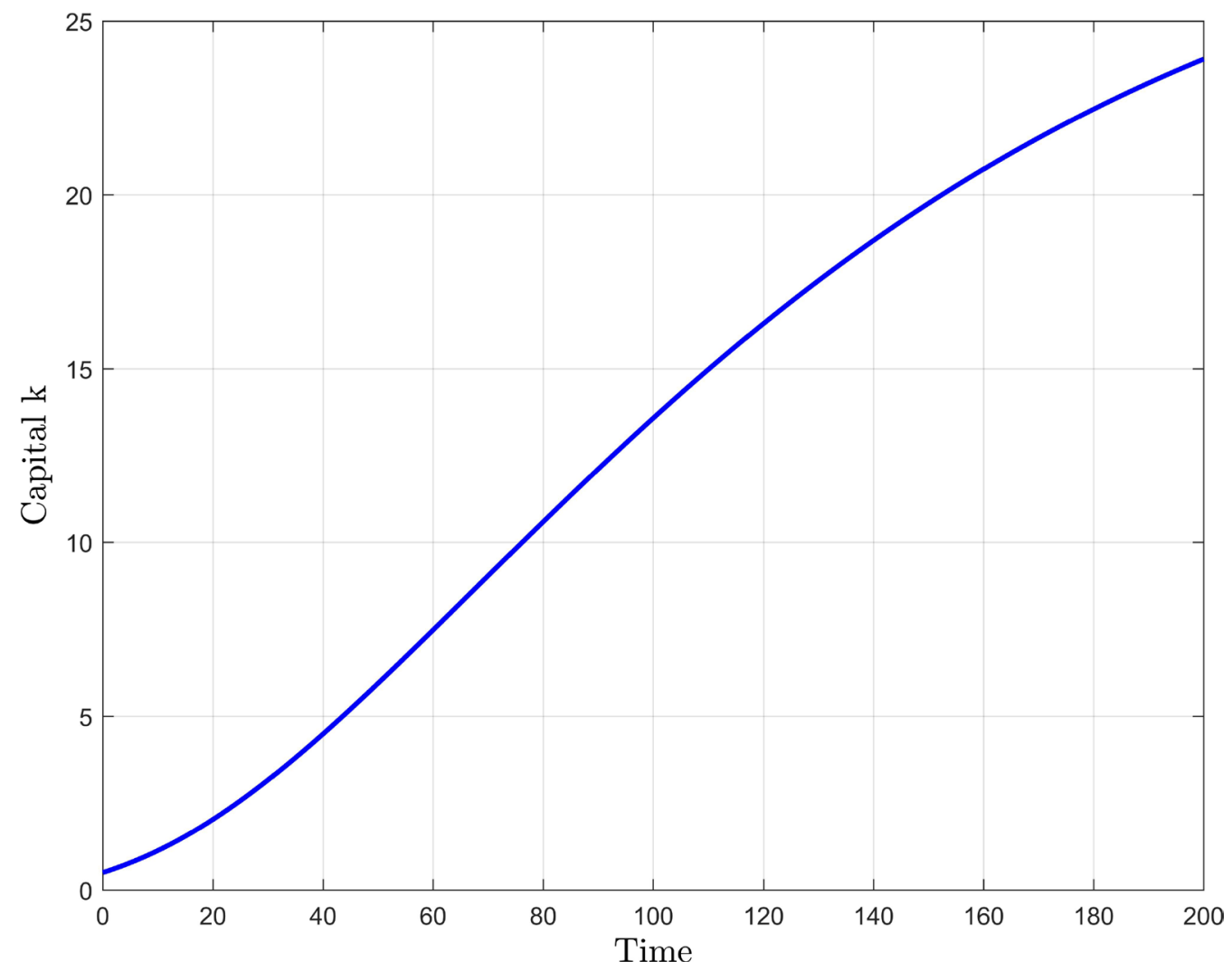

Figure 3. The path of capital, $k$.

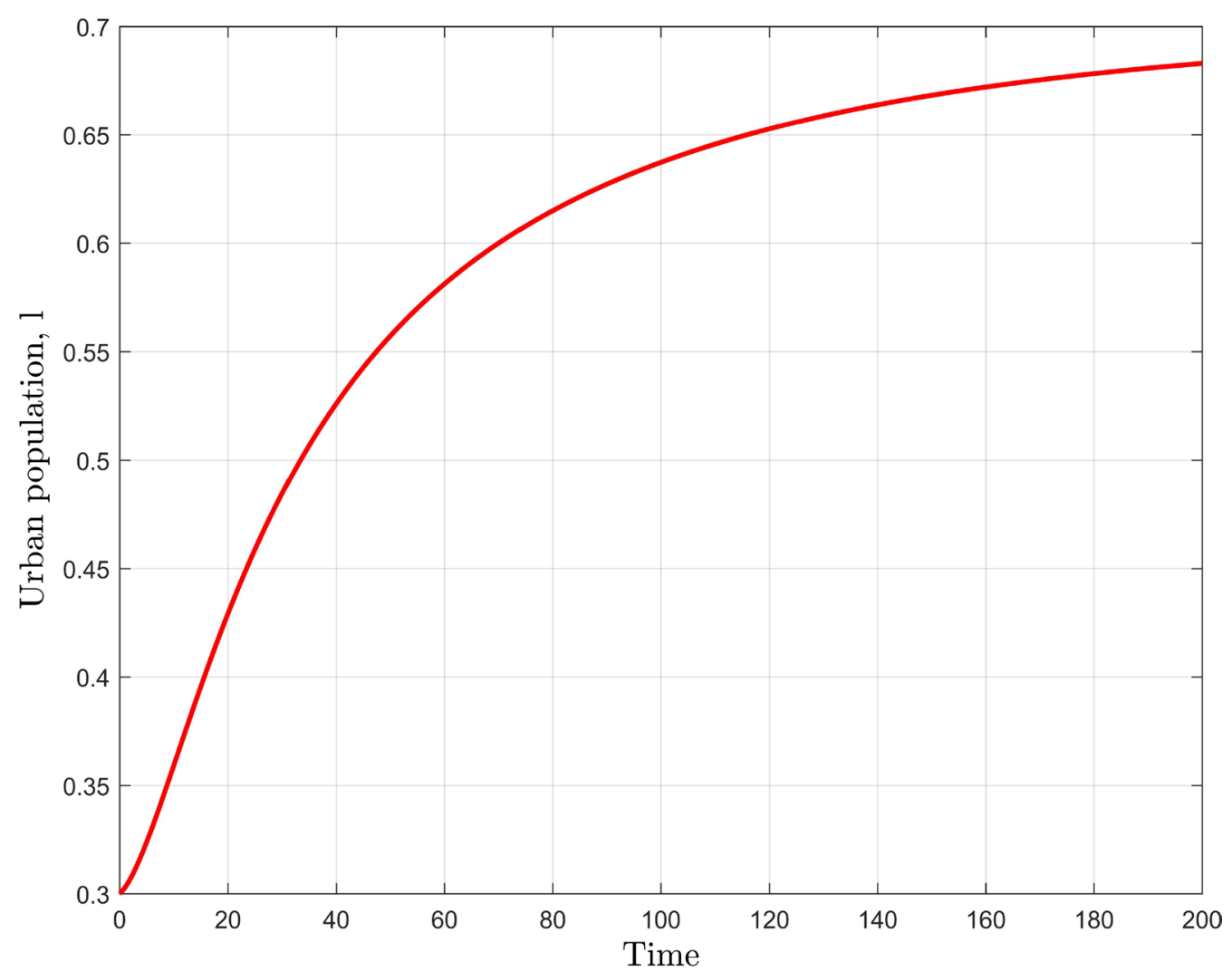

Figure 4. The evolution of urban population, $l$. 


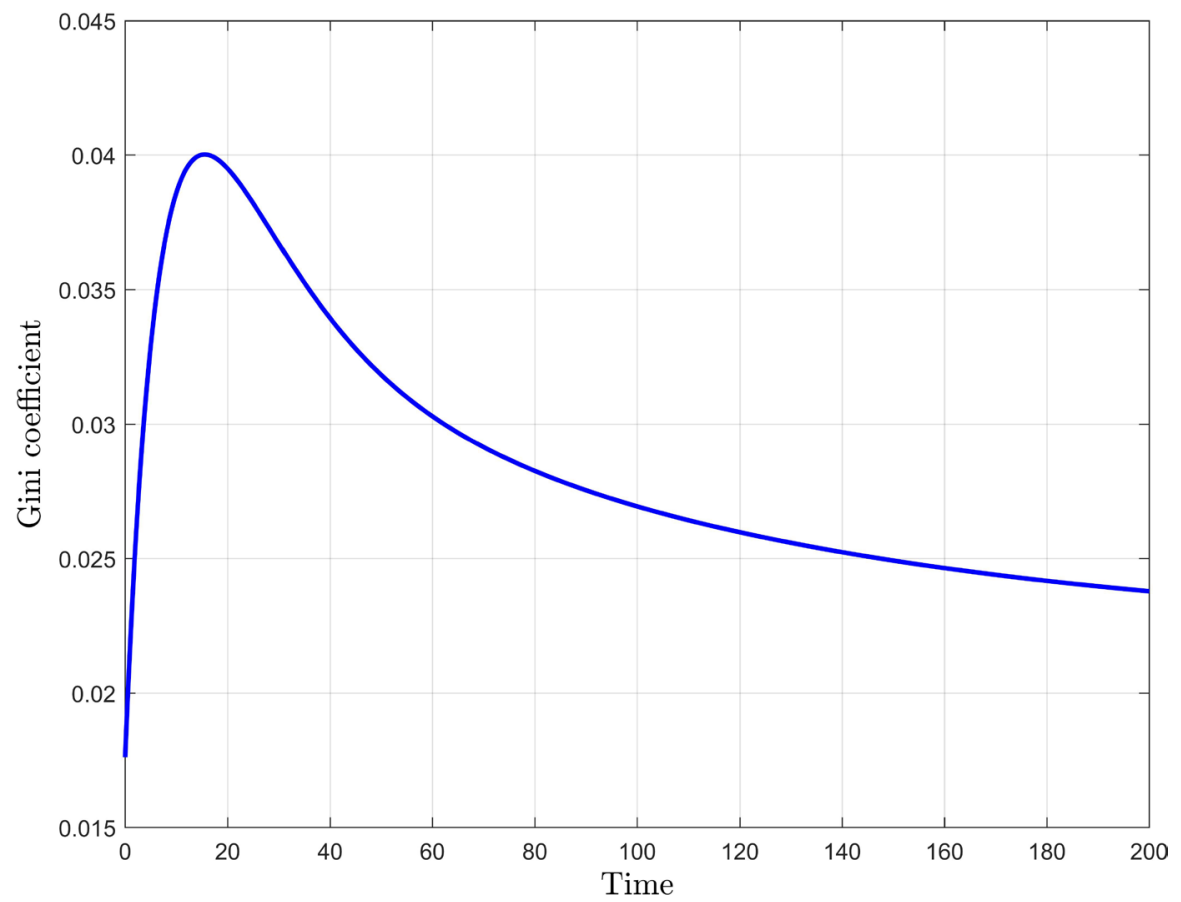

Figure 5. The evolution of Gini coefficient.

steady state to be achieved to be similarly long. (However, by specifying different initial conditions and parameter values to those specified here, it would be possible to reduce the time required for the steady state to be achieved in our model and this question is being explored in a separate paper).

\section{Parameter Values, Feasibility Regions, and Steady State Solutions}

In this section we examine how the effects of changes in the parameter values can be studied in a convenient way within the framework presented here. The fact that the values of all of these parameters can be influenced by government policies makes this exercise particularly interesting. The parameter $n$ (the rate of population growth) can clearly be influenced by government policies. In the introduction and in the preceding section, we referred to the parameters $\gamma$ and $\beta$ as reflecting the demand for labour in the informal and rural sectors, respectively. If technical progress in the rural sector is labour augmenting (as indeed the green revolution technology, which made double and multiple cropping possible, had been), then this would, ceteris paribus, tend to increase the demand for labour in the rural sector. ${ }^{11}$ Whether technical progress in the rural sector is labour augmenting or not can be influenced by the government via the nature and extent of its extension services, its factor pricing policies and its $\mathrm{R} \&$ $\mathrm{D}$ activities. ${ }^{12}$ Similarly, the value of the parameter $\gamma$ can be influenced by poli-

\footnotetext{
${ }^{11} \mathrm{We}$ are assuming that the demand for final output rises with economic development.

${ }^{12}$ As is well known, agricultural technology, unlike industrial technology, cannot be borrowed wholesale from abroad. It has to a great extent to be indigenously developed to suit local soil and climatic conditions, etc.
} 
cies relating to land use, credit facilities, property rights and those bearing on the sub-contracting relationships between the formal and informal sector firms in the urban area. The value of the parameter $\alpha$ can be influenced through trade union and labour legislation.

In examining the effects of changes in these parameter values, a convenient way of proceeding would be to show how changing the values of any two of the parameters, while holding the values of the other parameters unchanged, would affect the steady state solutions. A map of feasible and unfeasible regions can then be provided for each case. There are, of course, 15 such cases which can be considered. ${ }^{13}$ Here we concentrate on considering the case of changes in $\alpha$ and $\beta$. Results are shown in Figures 6-9. A feasibility map for $\alpha$ and $\beta$ values is provided in Figure 10. The Gini coefficient sensitivity analysis is provided in Figure 11 and Figure 12. Results showing the effects of changes in the other combinations of parameter values can be similarly presented. They are not presented here to save space, but are available in [6]. However, for the interested reader we present below a summary of some of the findings.

It is seen that the parameters $F_{m}$ and $\delta$ do not play any role in determining the stationary values of the variables $v^{*}, k, l$ and $v . F_{m}$ controls the rate of migration but is not related to the stationary values. $\delta$ appears in the exponential term of the integral and therefore controls how fast a (quasi) steady-state

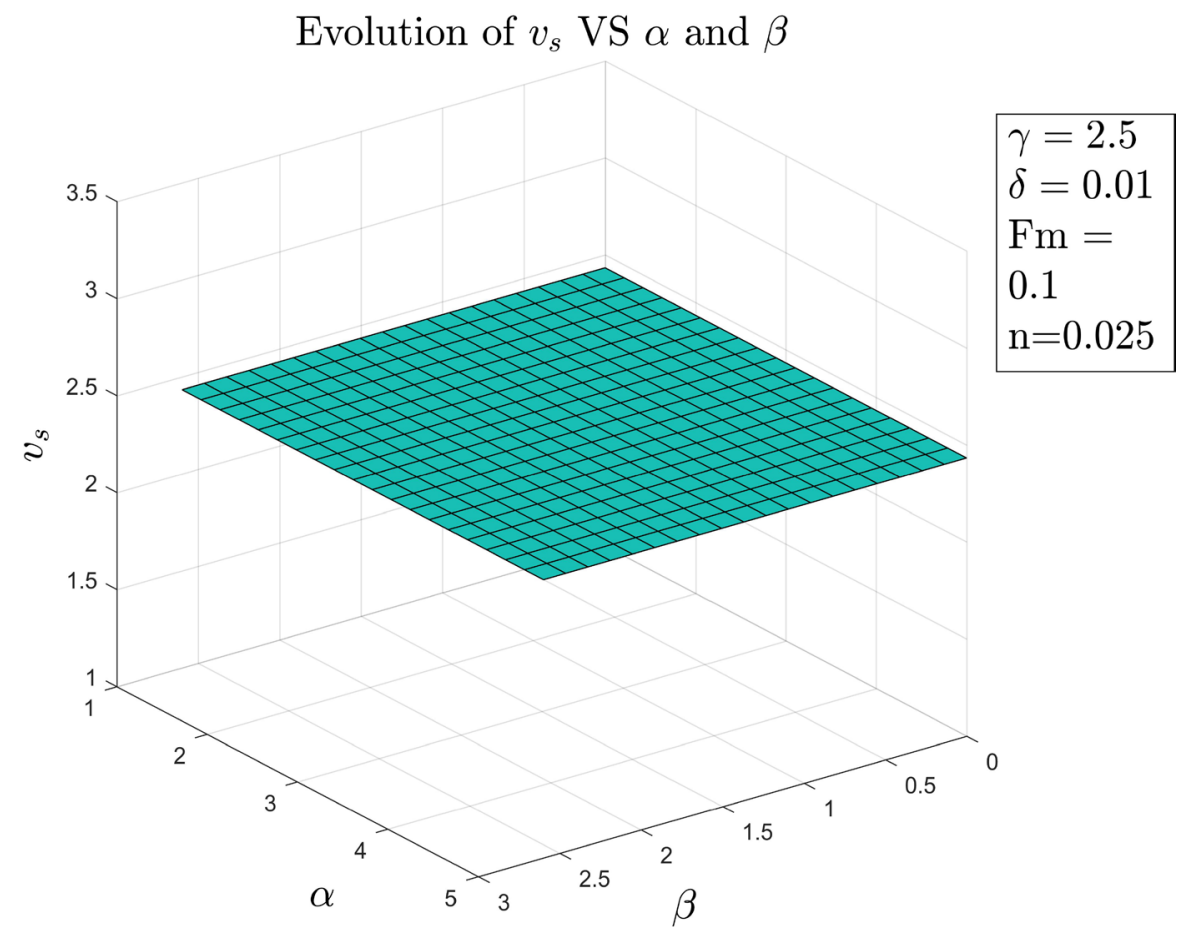

Figure 6. Evolution of $v^{*}$ vis-a-vis $\alpha$ and $\beta$. The steady state value of $v^{*}$ is only dictated by $n$, therefore, no changes occur when $\alpha$ and $\beta$ are modified.

\footnotetext{
${ }^{13}$ The cases are: changes in $\alpha$ and $\beta ; \alpha$ and $\gamma ; \alpha$ and $F_{m} ; \alpha$ and $n ; \alpha$ and $\delta ; \beta$ and $\gamma ; \beta$ and $n ; \beta$ and $F_{m} ; \beta$ and $\delta ; \gamma$ and $F_{m} ; \gamma$ and $n ; \gamma$ and $\delta ; F_{m}$ and $n ; F_{m}$ and $\delta$; and $n$ and $\delta$.
} 


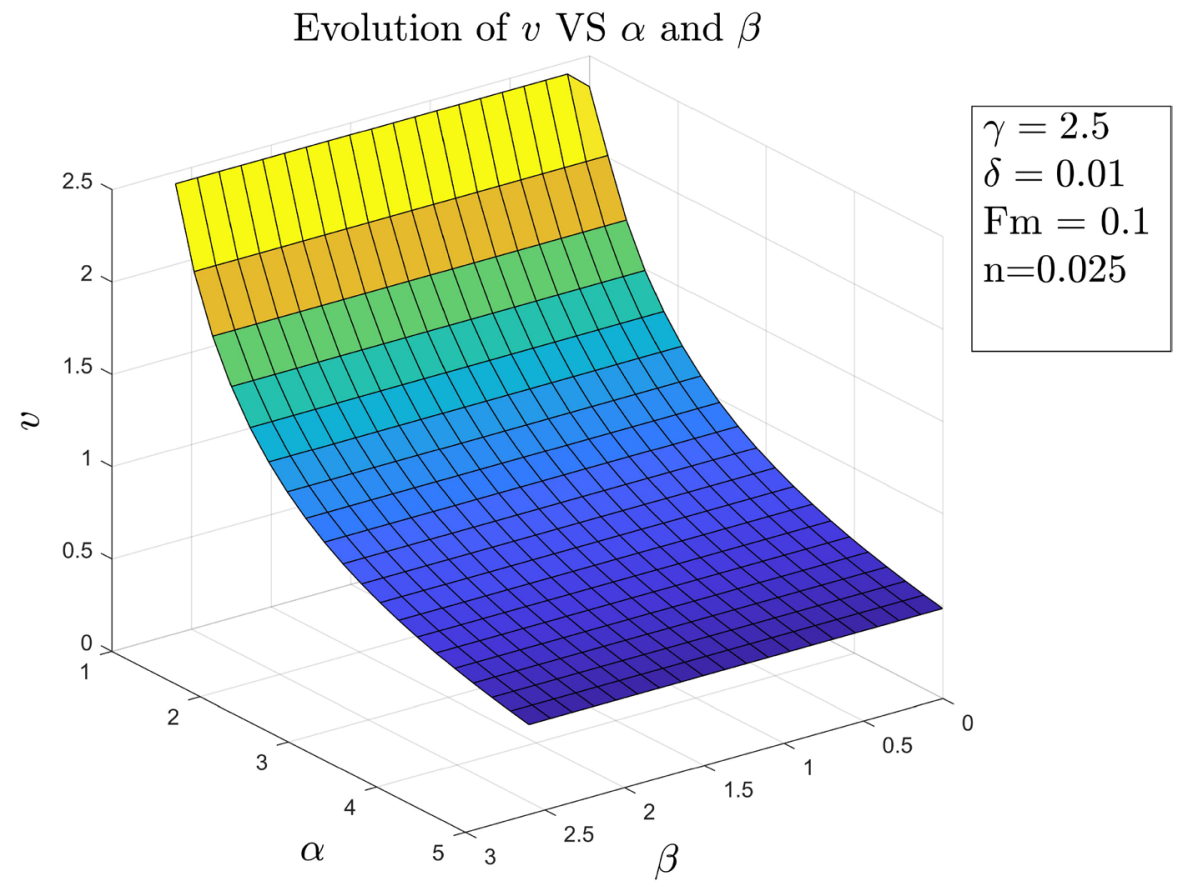

Figure 7. $\beta$ exerts little influence on the steady-state values of $v$ for large values of $\alpha$. For smaller values, when $\beta$ tends to zero, the value of $v$ is dependent on $\gamma, \alpha$ and $n$. A non-linear trend appears in that region. On the other hand, $\alpha$ has a greater dominance on the behaviour of $v$, which decreases nonlinearly as $\alpha$ increases. Varying $\beta$ causes small changes in $v$, whereas $\alpha$ has a strong and nonlinear influence. Particularly when $\alpha$ decreases, $v$ increases.

\section{Evolution of $k_{s} \operatorname{VS} \alpha$ and $\beta$}

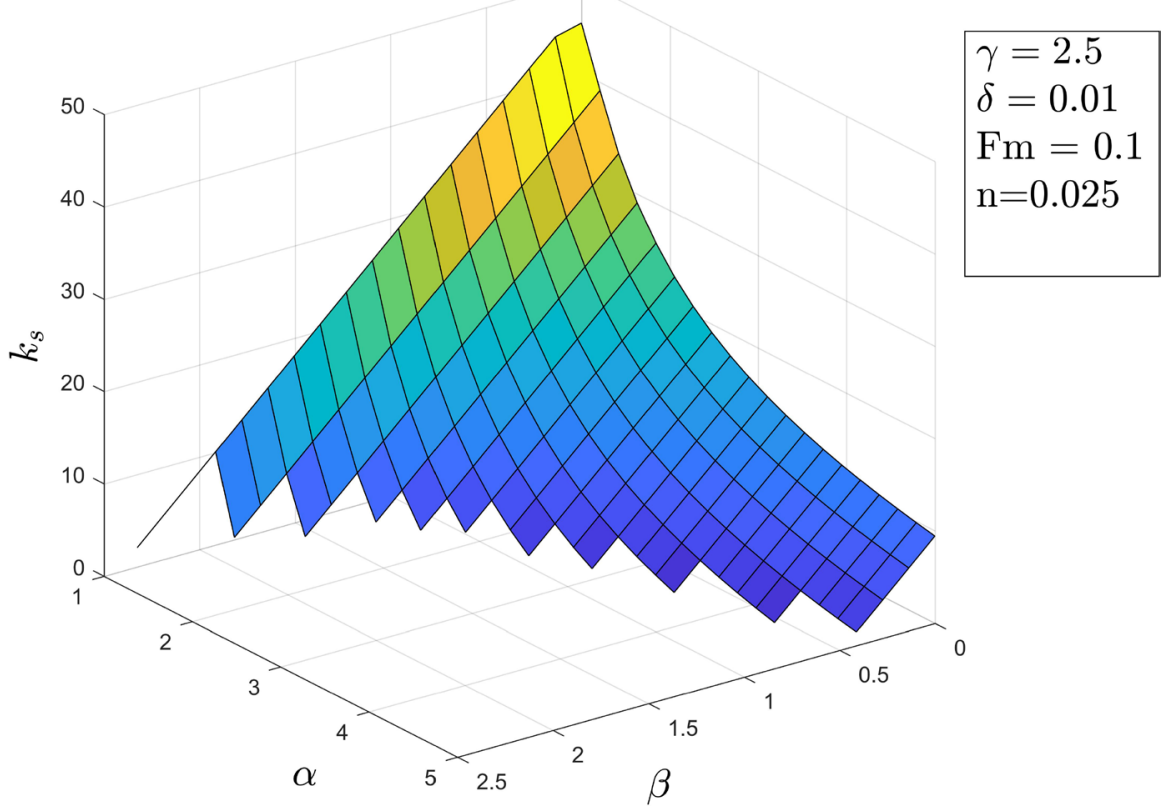

Figure 8. $k_{s}$ is a positive variable and therefore any negative values should be regarded as unfeasible. $k_{s}$ decays with increasing values of $\alpha$ and $\beta$ albeit with different trends. 


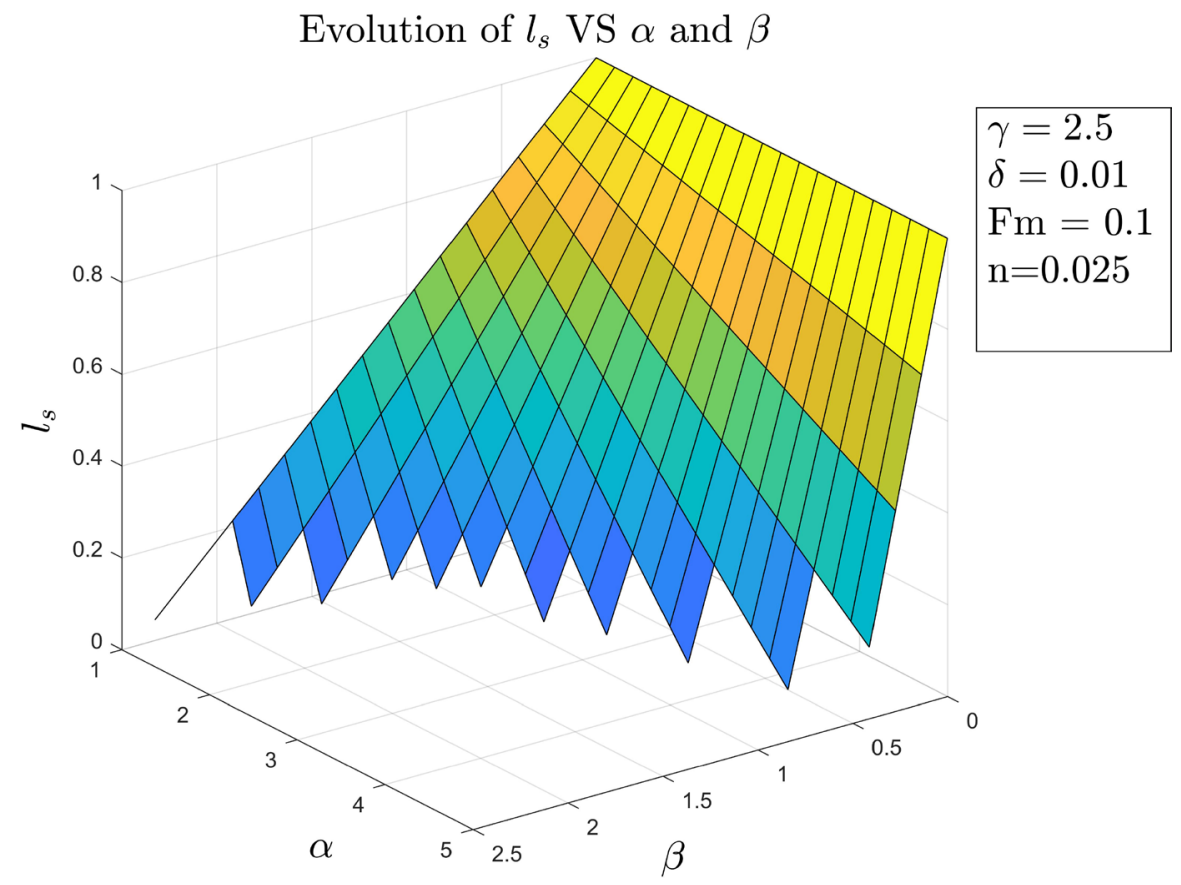

Figure 9. $l_{s}$ is a positive variable and therefore any negative values should be regarded as unfeasible. $l_{s}$ decays with increasing values of $\alpha$ and $\beta$ with quasi-linear trends.

Green: feasible. Blue: feasible $\left(v^{*}=\alpha v\right)$. Red: unfeasible

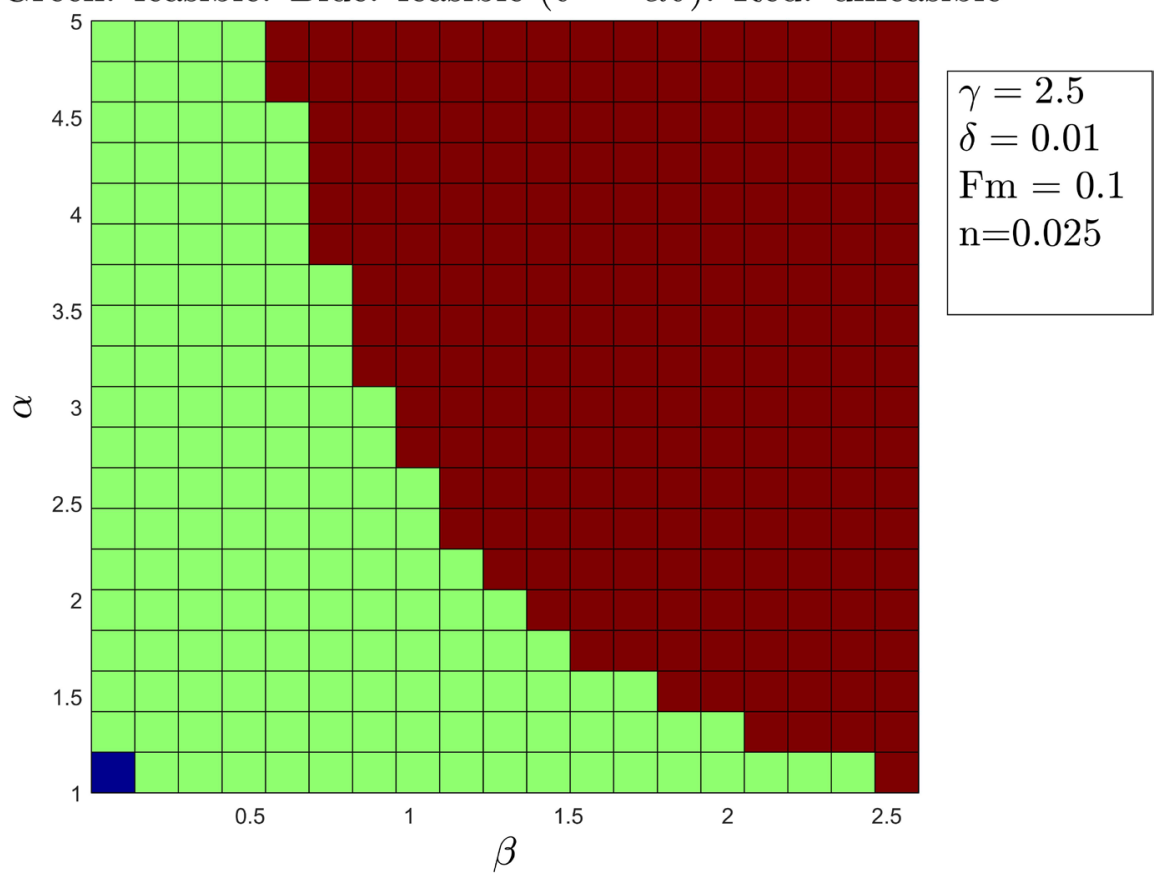

Figure 10. Feasibility map. The equality constraint $\left(v^{*}=\alpha v\right)$ is satisfied in most of the feasible region. Only very small values of $\beta$ and small values of $\alpha$ satisfy the inequality constraint. The feasible values of beta are greatly constrained for large values of $\alpha$. 


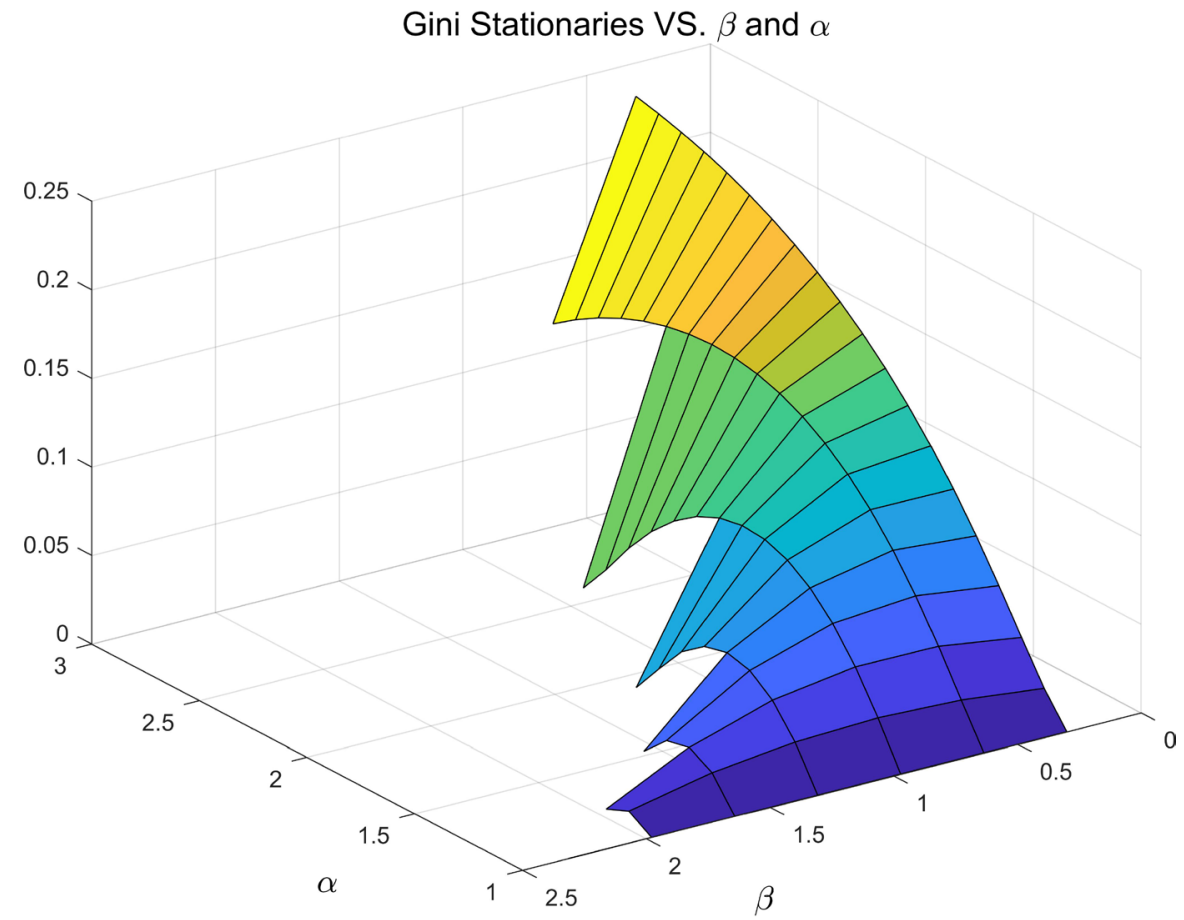

Figure 11. Steady state values of Gini coefficient when $\alpha$ and $\beta$ vary in the range of 1 to 3 and 0.3 to 5 respectively. Points not drawn represent unfeasible regions.

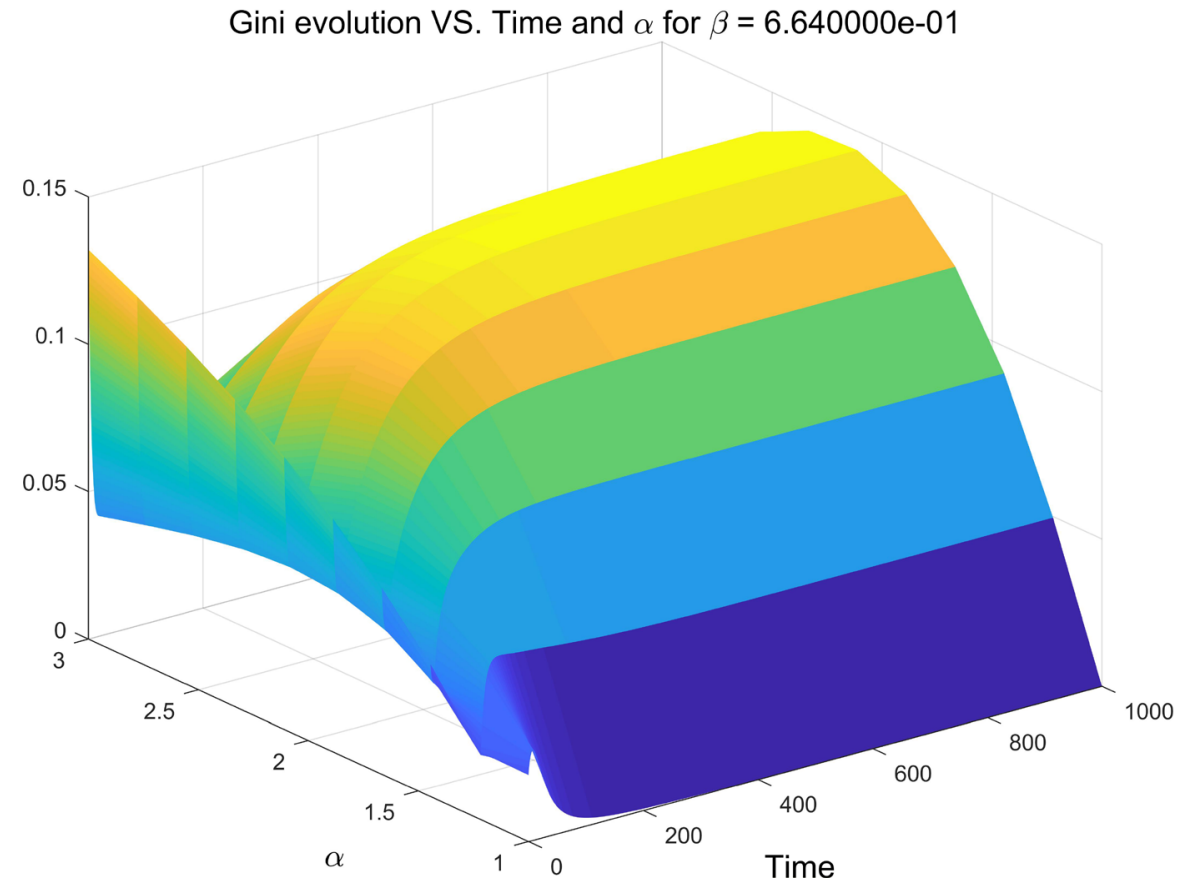

Figure 12. Gini coefficient decreases for low values of $\alpha$. The time to steady-state is also dependent on this parameter and is rapidly achieved, using this particular set of parameters, for $\alpha \approx 1.8$.

is achieved. No unfeasible regions are detected within a reasonable range of values for these parameters. 
The parameter $\gamma$ similarly does not play much of a role in determining the stationary values of $v^{*}, l$ and $v$. The stationary value of $k$, however, is influenced by $\gamma$. As $\gamma$ increases (decreases), $k$ decreases (increases).

As one would expect, the stationary value of $v^{*}$ depends only on $n$ and remains constant when varying any other parameters. The dependency on $n$ is like

$\frac{1}{\sqrt{n}}$, so a singularity can be observed when $\mathrm{n}$ tends to zero.

Finally, the stationary value of $k$ is seen to have a strong dependency on $\alpha$ with low values of $n$. The larger the $n$, the weaker that dependency is. Also $k$ grows fast when $\mathrm{n}$ is close to zero (whatever $\alpha$ is). Nevertheless, this happens most markedly when $\alpha$ closely approximates to one.

\section{Concluding Remarks}

In this paper we have presented a framework for thinking about optimal development of an economy with an informal sector. While we assumed a particular set of parameter values to derive our results in Section 3, in Section 4 we showed how the effects of changes in these parameter values can be studied in a convenient way within the framework presented here. We also derived the evolution of the Gini coefficient along the optimal path. We assumed a Cobb-Douglas production function and a particular utility function. It would be possible to relax these assumptions and work with other production and utility functions. The framework presented here-it is hoped-can be of some help in thinking about policies relating, inter alia, to population growth, inter-sectoral migration, agriculture-industry relationship, wages in different sectors, and income distribution in an inter-connected way in the context of optimal development of an economy with an informal sector.

A list of policy related questions that could be discussed could include the following. If the society, for example, wishes to have a particular distribution of the population between the urban and rural areas (or between the various sectors) at the steady state, what policies or combinations of policies would be best suited to achieve this and what would be the consequences of adopting these policies not only for, say, the steady state value of capital, but also for the evolution of income distribution along the optimal path? Similarly, if the society wishes the rural and urban wages to be equalized within a specified time period, what policies could be adopted to achieve this and what would be the consequences of adopting these policies for income distribution as well as the distribution of people between the urban and rural areas along the optimal path? What would be the effect of these policies on the steady state value of capital? How would any target growth rate of the population affect the evolution of the economy along the optimal path? How would any government policy to encourage the demand for labour in the informal sector influence the evolution of the economy along the optimal path and the steady state values of urban labour, capital, etc.? How would policies to encourage the development and adoption of labour augmenting tech- 
nical progress in the rural sector affect the time to steady state as well as the steady state values of the various variables? Clearly, one can think of other questions along similar lines.

\section{Conflict of Interest}

The author declares that there is no conflict of interest regarding the publication of this paper.

\section{References}

[1] Sen, A.K. (1960) Choice of Techniques: An Aspect of the Theory of Planned Economic Development. Basil Blackwell, Oxford.

[2] Dixit, A.K. (1968) Optimal Development in the Labour-Surplus Economy. Review of Economic Studies, 35, 23-34. https://doi.org/10.2307/2974404

[3] Stern, N.H. (1972) Optimum Development in a Dual Economy. Review of Economic Studies, 39, 171-184. https://doi.org/10.2307/2296869

[4] Kuznets, S. (1955) Economic Growth and Income Inequality. American Economic Review, 45, 1-28.

[5] Atkinson, A.B. (1969) The Timescale of Economic Models: How Long Is the Long Run? Review of Economic Studies, 36, 137-152. https://doi.org/10.2307/2296833

[6] Bhattacharya, P.C. (2015) A Model of Optimal Development. Heriot-Watt University Discussion Paper in Economics.

https://www.hw.ac.uk/schools/social-sciences/documents/HW_DP_2015_04.pdf

[7] Bhattacharya, P.C. (1994) A Multi-Sector Model of LDC. Scottish Journal of Political Economy, 41, 225-255. https://doi.org/10.1111/j.1467-9485.1994.tb01123.x

[8] Bhattacharya, P.C. (2011) Informal Sector, Income Inequality and Economic Development. Economic Modelling, 28, 820-830.

https://doi.org/10.1016/j.econmod.2010.10.007

[9] Bhattacharya, P.C. (1993) Rural-Urban Migration in Economic Development. Journal of Economic Survey, 7, 243-281. https://doi.org/10.1111/j.1467-6419.1993.tb00167.x

[10] Bhattacharya, P.C. (1998) The Informal Sector and Rural-to-Urban Migration: Some Indian Evidence. Economic and Political Weekly, 33 1255-1257, 1259-1262.

[11] Bhattacharya, P.C. (2002) Rural-to-Urban Migration in LDCS: A Test of Two Rival Models. Journal of International Development, 14, 951-972. https://doi.org/10.1002/jid.915

[12] Todaro, M.P. (1969) A Model of Labor Migration and Urban Unemployment in Less Developed Countries. American Economic Review, 59, 138-148.

[13] Harris, J.R. and Todaro, M.P. (1970) Migration, Unemployment and Development: A Two-Sector Analysis. American Economic Review, 60, 126-142.

[14] Williamson, J.G. (1988) Migration and Urbanization, In: Chenery, H. and Srinivasan, T.N., Eds., Handbook of Development Economics, North-Holland, Amsterdam, 425-465.

[15] Anand, S. and Kanbur, S.M.R. (1993) The Kuznets Process and the Inequality-Development Relationship. Journal of Development Economic, 40, 25-52.

[16] Alesina, A. and Rodrik, D. (1994) Distributive Politics and Economic Growth. Quarterly Journal of Economics, 109, 465-490. https://doi.org/10.2307/2118470 
[17] Barro, R.J. (2000) Inequality and Growth in a Panel of Countries. Journal of Economic Growth, 5, 5-32. https://doi.org/10.1023/A:1009850119329

[18] Forbes, K.J. (2000) A Reassessment of the Relationship between Inequality and Growth. American Economic Review, 90, 869-887.

https://doi.org/10.1257/aer.90.4.869

Submit or recommend next manuscript to SCIRP and we will provide best service for you:

Accepting pre-submission inquiries through Email, Facebook, LinkedIn, Twitter, etc. A wide selection of journals (inclusive of 9 subjects, more than 200 journals)

Providing 24-hour high-quality service

User-friendly online submission system

Fair and swift peer-review system

Efficient typesetting and proofreading procedure

Display of the result of downloads and visits, as well as the number of cited articles Maximum dissemination of your research work

Submit your manuscript at: http://papersubmission.scirp.org/

Or contact jamp@scirp.org 